\title{
The bactericidal effect of liposomal vancomycin as a topical combating system against Methicillin-resistant Staphylococcus aureus skin wound infection in mice
}

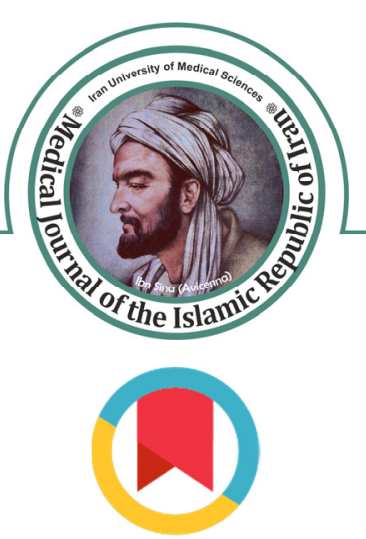

\author{
Fahimeh Hajiahmadi ${ }^{1}$, Mohammad Yousef Alikhani ${ }^{1}$, Hanifeh Shariatifar ${ }^{2}$, Mohammad Reza Arabestani*1,3, \\ Davoud Ahmadvand*4,5
}

Received: 7 May 2019

Published: 26 Dec 2019

\section{Abstract}

Background: Methicillin-resistant Staphylococcus aureus (MRSA) is one of the most common causes of skin infections and treatment is difficult due to its resistance to the most of antibiotics. Although vancomycin is often considered as an antibacterial agent of choice for the treatment of MRSA, its use is limited because of the high side effects. One solution is using liposomal formulation for local drug delivery. The aim of this study was to determine in vitro and in vivo efficacies of liposomal vancomycin as topical use.

Methods: To prepare liposomal vancomycin, the ammonium sulfate gradient using remote loading and freeze-thaw methods was applied. Then, synthesized nanoliposomes were evaluated in terms of particle size, morphology, stability, and encapsulation efficiency. Minimum inhibitory concentration (MIC) of synthesized nanoliposome against MRSA was detected. The cytotoxicity of synthesized nanoliposome was evaluated using MTT (3-(4,5-Dimethylthiazol-2-yl)-2,5-diphenyltetrazolium bromide) assay. Finally, the topical antibacterial activity of each formulation was tested against MRSA-infected skin wound model in mice.

Results: High encapsulation efficiency was achieved for all synthesized nanoliposomes. The results of in vitro and in vivo showed that liposomal vancomycin was more effective than free vancomycin. Also, synthesized nanoliposome showed no cytotoxicity on human epidermoid cell line.

Conclusion: The results showed that synthesized nanoliposome could be applied as a great topical antimicrobial construct for treatment of MRSA skin infections.

Keywords: MRSA, Antibacterial activity, Vancomycin, Liposome

Conflicts of Interest: None declared

Funding: This work was supported by a research grant from Hamadan University of Medical Sciences (Grant/Award Number: 94-10-29-6134) and Iran University of Medical Sciences (Grant/ Award Number: 94-05-31-26873).

\section{*This work has been published under CC BY-NC-SA 1.0 license.}

Copyright $($ Iran University of Medical Sciences

Cite this article as: Hajiahmadi F, Alikhani MY, Shariatifar H, Naseri A, Arabestani MR, Ahmadvand D. The bactericidal effect of liposomal vancomycin as a topical combating system against Methicillin-resistant Staphylococcus aureus skin wound infection in mice. Med J Islam Repub Iran 2019 (26 Dec);33:153. https://doi.org/10.47176/mjiri.33.153

\section{Introduction}

Staphylococcus aureus is one of the most common causes of skin infections (1), and an increased prevalence of methicillin-resistant $S$. aureus (MRSA) has been observed in the hospital and in the community setting (2). For many years, conventional antibiotics such as penicillin were employed

Corresponding author: Dr Mohammad Reza Arabestani,mr.arabestani@umsha.ac.ir Dr Davoud Ahmadvand, d.ahmadvand@iums.ac.ir

1. Department of Microbiology, Hamadan University of Medical Sciences, Hamadan, Iran

2. Young Researches and Elite Club, Tehran Medical Sciences, Islamic Azad University, Tehran, Iran

3. Brucellosis Research Center, Faculty of Medicine, Hamadan University of Medical Sciences, Hamedan, Iran

4. Department of Medical Laboratory Sciences, Faculty of Allied Medicine, Iran University of Medical Sciences, Tehran, Iran

5. Neuroscience Research Center, Iran University of Medical Sciences, Tehran, Iran against $S$. aureus infections. However, MRSA isolates are now resistant to the most $ß$-lactam antibiotics which are considered as a global problem. Vancomycin has been the first-line drug in the treatment of MRSA infect ions (3). Nevertheless, reduced susceptibility to this drug has

$\uparrow$ What is "already known" in this topic:

MRSA is one of the most common pathogens in postsurgical wound. Vancomycin is the drug of choice for treating most MRSA infections. Unfortunately, its use is limited due to the side effects of systemic vancomycin treatment.

\section{$\rightarrow$ What this article adds:}

In this study, liposomal vancomycin was used as a topical form. Vancomycin could be selectively delivered by liposomes to the infected site to reduce site effects and increase drug potency locally. 
emerged (4). The side effects of systemic vancomycin treatment, including allergic reactions, nephrotoxicity, ototoxicity, neuromuscular blockade, and patient discomfort, may exert a huge influence on the efficiency of treatment (5). Also, the rate of initial treatment failure is increasing due to the emergence of resistant bacteria in skin infections (6). Therefore, there is an urgent need to reduce its side effects and develop better therapies. One solution is to discover new, safe, and effective topical liposomal vancomycin. Alternatively, vancomycin could be selectively delivered by liposomes to the infected site to reduce site effects and increase drug potency locally. Liposomes have been used as intravenous administration in most experimental studies, while in this study, liposomal vancomycin was used as a topical form. For decades, liposomes have been used as a drug delivery vehicle. Their advantages are as follow: being composed of biocompatible lipid materials; the ability to deliver hydrophilic and lipophilic drugs; the ability to fuse with bacteria cell membranes; easy surface modification (7); and the ability to concentrate at the target site due to the increased permeability and maintenance effect (8). The combination of topical liposomal vancomycin provides a potentially powerful means of improving vancomycin delivery. The aim of this study was to determine in vitro and in vivo efficacies of liposomal vancomycin for topical use.

\section{Methods \\ Materials}

L- $\alpha$-phosphatidylcholine (Egg-PC) was purchased from Avanti Polar Lipids, Inc (AL, USA). Cholesterol, vancomycin hydrochloride, methylthiazolyldiphenyl-tetrazolium bromide (MTT), dimethyl sulfoxide (DMSO), penicillin, streptomycin, RPMI-1640 medium, Triton X-100, and sodium chloride were from Sigma Aldrich (MO, USA). Blood agar, mueller hinton (MH) broth, methanol, chloroform, and ammonium sulfate were obtained from Merck (Darmstadt, Germany). Sepharose CL-4B was purchased from GE Health care (Bucks, UK). Antibiotic disks were purchased from MAST (Merseyside, UK). Gene MATRIX Quick Blood DNA Purification Kit was purchased from EURx Ltd (Gdańsk, Poland). All animal experiments were done in accordance with the protocols approved by the Ethics Review Body of Animal Experimentation of Iran University (IR.UMSHA.REC.1394.134).

\section{Bacterial strains}

A clinical isolate of $S$. aureus was isolated from a patient with a skin wound infection (Hamadan Hospital, Iran) and confirmed by conventional biochemical tests and the presence of the femA gene (9). S. aureus ATCC 25923 was used as a control strain. Bacteria were stored at $-70^{\circ} \mathrm{C}$ in MH broth supplemented with $20 \%$ (v/v) glycerol. Template DNA was extracted using GeneMATRIX Quick Blood DNA Purification Kit. Presence of the $m e c A$ gene was detected by PCR using specific primers, as previously described (10).

\section{Antibiotics and susceptibility testing}

S. aureus strain was tested for antibiotic susceptibility by an agar dilution method and a broth dilution method according to the Clinical and Laboratory Standards Institute (CLSI) guidelines (11). The antibiotics included cefoxitin $(30 \mu \mathrm{g})$, norfloxacin $(10 \mu \mathrm{g})$, tobramycin $(10 \mu \mathrm{g})$, ciprofloxacin $(5 \mu \mathrm{g})$, gatifloxacin $(5 \mu \mathrm{g})$, amikacin $(30 \mu \mathrm{g})$, linezolid $(30 \mu \mathrm{g})$, rifampin $(5 \mu \mathrm{g})$, doxycycline $(30 \mu \mathrm{g})$, oxacillin, and vancomycin.

\section{Preparation of liposomal vancomycin}

Formulations of liposomal vancomycin were prepared using 2 methods:

Remote loading method by ammonium sulfate gradient: Liposomes were prepared by the thin-film hydration method in a molar ratio of 6.5/3.5 (Egg -PC / Cholesterol) (50 mg). The lipids were dissolved in 9:1 (v/v) chloroform and methanol; then, the lipid film was hydrated in ammonium sulfate solution $(250 \mathrm{mM}, \mathrm{pH} 5.5)$. Following vigorous vortexing for 5 minutes, the multilamellar vesicles (MLVs) were sonicated using a probe sonicator for 3 minutes (pulsed duty cycle of 45 seconds on and 15 seconds off, power delivery of $30 \%$ ) (Vibra-Cell TM Jencons Scientific Ltd., USA). The solution was extruded through a $200 \mathrm{~nm}$ pore-sized polycarbonate membrane 21 times at room temperature (RT) to make small unilamellar vesicles (SUVs) (Avanti Polar Lipids, USA). Next, liposome solution and vancomycin solution $(1 \mathrm{~mL}$ of $300 \mathrm{mM} \mathrm{NaCl}$ with $\mathrm{pH} 5.5$ containing $10 \mathrm{mg}$ of vancomycin) were mixed and incubated for 2 hours at $4{ }^{\circ} \mathrm{C}$. The nonencapsulated vancomycin was separated using a Sepharose CL-4B gel filtration column $(24 \times 1 \mathrm{~cm}$ internal diameter). Vancomycin encapsulation efficiency was surveyed with adding $200 \mu \mathrm{L}$ of $10 \%$ Triton X-100 to $90 \mu \mathrm{L}$ of prepared liposome and incubated at $37^{\circ} \mathrm{C}$ for 5 minutes; then, free vancomycin was measured by HPLC analysis (Knauer, Italy) (12).

Freeze-thaw method: A lipid film was prepared using 50 mg of the lipids Egg-PC: cholesterol, in a molar ratio of $6.5 / 3.5$. The lipid film was hydrated using $1.5 \mathrm{~mL}$ of phosphate-buffered saline (PBS) (pH: 7.4) containing $10 \mathrm{mg}$ of vancomycin. The rehydrated film was vortexed, sonicated, and extruded as described above. Then, the mixture was frozen at $-20^{\circ} \mathrm{C}$ for 20 minutes and at $45^{\circ} \mathrm{C}$ for 10 minutes by 3 freeze-thaw cycles. Sepharose CL-4B gel filtration column $(24 \times 1 \mathrm{~cm}$ internal diameter $)$ was used to separate the unloaded vancomycin and measure its concentration by HPLC (13).

\section{Liposome characterization}

The particle size distribution and polydispersity index (PDI) were determined on a Nano-flex $180^{\circ}$ dynamic light scattering (DLS) analysis (Particle Metrix, Germany) at $25^{\circ} \mathrm{C}$ with refractive index 1.3332 , viscosity of 0.8878 , and scattering intensity of 11480 . Stability of prepared liposomes was evaluated in terms of physical properties and the amount of vancomycin released by 6 months at $4^{\circ} \mathrm{C}$ and $25^{\circ} \mathrm{C}$.

\section{Antimicrobial susceptibility test}

The MIC (minimal inhibitory concentration) of free vancomycin and liposomal vancomycin was evaluated using a liquid growth inhibition assay in 96 well microtiter plates 
as recommended by CLSI guidelines (11). MIC was considered as the lowest concentration of antibiotic that completely inhibited the visible growth of bacteria after 16-18 hours of incubation at $35^{\circ} \mathrm{C}$.

\section{Transmission electron microscopy}

Transmission electron microscopy (TEM) was done on a Zeiss-EM10C instrument (Germany) at an acceleration voltage of $100 \mathrm{Kv}$. Briefly, freshly prepared liposomal formulations were placed on a 200-mesh formvar copper grid (TABB Laboratories Equipment, Berks, UK). Negative staining was performed by adding a drop of $2 \%(\mathrm{w} / \mathrm{v})$ aqueous solution of uranyl acetate ( 5 minutes) to the copper grid with the sample. Then, the samples were dried at $25^{\circ} \mathrm{C}$ before the vesicles were analyzed by TEM.

\section{Cytotoxicity Test}

In vitro cytotoxicity of free vancomycin and liposomal vancomycin was measured using MTT (3-(4,5-Dimethylthiazol-2-yl)-2,5-diphenyltetrazolium bromide) assay. In this study, human epidermoid carcinoma epithelial cell line (A431; ATCC ${ }^{\circledR}$ CRL-1555 ${ }^{\mathrm{TM}}$ ) was used for cell toxicity assay. Briefly, the cells were seeded in 96 well plates at a density of $5 \times 10^{3}$ cells/well for subconfluency and incubated for 24 hours at $37^{\circ} \mathrm{C}$. The next day, cells were treated with all formulations and the untreated cells were considered as controls. After a 24-hour incubation, the cells were washed twice with Dulbecco's PBS and replaced with fresh medium. MTT dye was added to each well and incubated for 4 hours. Finally, dimethyl sulfoxide was added and the absorbance at $570 \mathrm{~nm}$ was measured using a microplate reader (Bio-Rad; Model 680) to calculate cell viability.

\section{Mice and bacteria culture}

The MRSA culture was prepared one day before infection in accordance with Wen et al study (14). Male NMRI mice aged between 6 and 8 weeks were used for this study. All mice were put in separate cages to avoid fighting and given sterile chow and water ad libitum. All the cages were changed and autoclaved to prevent secondary bacterial contamination, and mice were kept on a 12-h light dark cycle at RT for a week to adapt to the environment (15)._Initially, the MRSA doses leading to mortality in a murine model of the surgical wound were determined. A region of the skin was excised by biopsy punch and the wound was infected with various colony-forming unit (CFU) counts of MRSA, including $10^{7}, 10^{8}$ and $10^{9}$. Three groups of mice (9 mice in each group) were used in this experiment: (1) mice given bacteria and liposomal vancomycin simultaneously, (2) mice given bacteria and free vancomycin, (3) and mice treated with PBS and liposome without bacteria. After shaving the hair on the back of the mice, a full-thickness wound was created by biopsy punch $(7 \mathrm{~mm})$, and each wound was inoculated with $10 \mu \mathrm{L}$ of broth containing $10^{7}$ CFU of MRSA. Treatment was done based on the MIC dose. A single daily dose of liposomal vancomycin containing $3 \mu \mathrm{g}$ of vancomycin was as effective as 2 daily doses of the $3 \mu \mathrm{g}$ of free vancomycin.

Nonliposomal drugs were administered every 12 hours. However, liposomal drugs were used as a single daily dose. On the Forth, ninth, and 14th days, mice were anesthetized and sacrificed. After homogenizing lesion skin specimens, the bacteria were counted. Furthermore, spleen and liver were collected, homogenized, and counted for bacterial number.

\section{Statistical analysis}

The data were analyzed with SSPS version 20.0 software (SPSS). Significant differences were detected between groups $(\mathrm{p}<0.05)$ using ANOVA and Tukey test.

\section{Results}

\section{Antimicrobial susceptibility test}

$S$. aureus strain isolated from the wound was confirmed through the presence of the fem $A$ gene (Fig. 1a). The results of antimicrobial susceptibility testing showed that $S$. aureus was resistant to most antibiotics, including cefoxitin, oxacillin, norfloxacin, ciprofloxacin, gatifloxacin, and rifampin. Also, it was considered as a multidrug resistant (MDR) strain. On the other hand, the lowest level of resistance was observed against aminoglycosides, linezolid, doxycycline, and vancomycin. The results of PCR analysis showed presence of mecA gene and verified resistance to methicillin (Fig. 1b). a

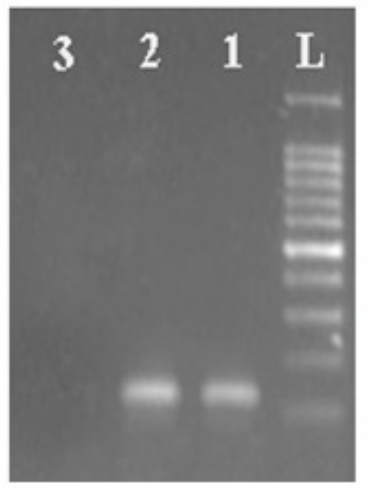

b

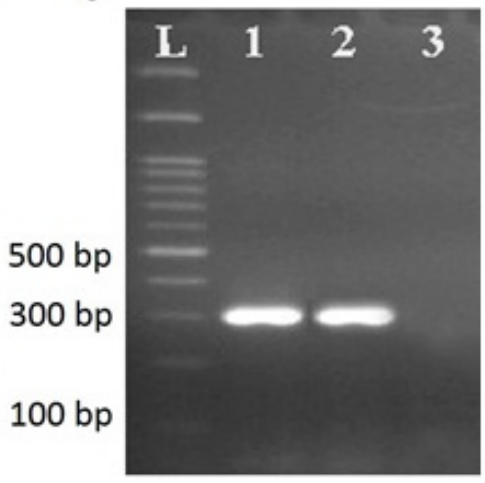

Fig. 1. Agarose gel electrophoresis for the detection of the femA gene (132 bp) (a) and mecA gene (297 bp) (b) in MRSA clinical isolate by PCR. Lanes: L, molecular marker (100 bp DNA ladder); 1, Staphylococcus aureus ATCC 33591 as positive control; 2 , MRSA clinical isolate; and 3, E. coli ATCC 25922 as negative control. 


\section{Liposome characterization}

Particle size: Both the liposomal formulations prepared by ammonium sulfate gradient and freeze-thaw methods have shown a mean particle size of $193 \pm 2.1 \mathrm{~nm}$ and 178 $\pm 5.5 \mathrm{~nm}$, respectively (Table 1). Particle sizes of prepared liposomes by freeze-thaw were slightly smaller than of prepared liposomes by ammonium sulfate gradient. The PDIs of PDIs of the liposomes was less than 0.2 , indicating a relatively homogenous size distribution.

Encapsulation efficiency: High entrapment of vancomycin was achieved in both methods. There was a substantial improvement in encapsulation efficiency when the freezethaw method was applied compared to the ammonium sulfate gradient method (Table 1).

In vitro drug release of liposomes: The stability of all liposomes was evaluated after 6 months in PBS at $4^{\circ} \mathrm{C}$ and $25^{\circ} \mathrm{C}$ (Fig. 2). No aggregation was observed in any of the preparation. The released vancomycin from prepared liposomes by ammonium sulfate gradient was slightly higher than of prepared liposomes by freeze-thaw in both $4^{\circ} \mathrm{C}$ and $25^{\circ} \mathrm{C}$.
Morphology of liposomes: Negatively stained transmission electron was applied to show the morphology of all liposomes (Fig. 3). The TEM images indicated that most liposomes were spherical in both ammoniums sulfate gradient and freeze-thaw methods. The synthesized liposomes by the ammonium sulfate method had the uniform dispersion without aggregation, whereas in the freeze-thaw method, the vesicles were discrete in distribution.

Liposomal antimicrobial activity: Antimicrobial activities of free vancomycin and liposomal vancomycin against MRSA and S. aureus (ATCC 25923) are illustrated in Table 2 . The results showed that liposomal vancomycin was more effective than free vancomycin.

Cell viability assay: The results of cell viability assay using MTT dye showed that free vancomycin led to a reduction in cell viability compared to liposomal vancomycin, indicating that the cytotoxicity of the vancomycin was very limited when incorporated in liposome $(\mathrm{p}<0.05)$. Vancomycin up to $\leq 18.7 \mathrm{ug} / \mathrm{mL}$ showed no toxicity after 24 hours of treatment. However, liposomal vancomycin showed no toxicity up to $\leq 75 \mathrm{ug} / \mathrm{mL}$ (Fig. 4), indicating that liposomal vancomycin and free vancomycin were noncytotoxic $6 \times$

Table 1. Physicochemical parameters of liposomes

\begin{tabular}{lcc}
\hline Methods & Ammonium sulfate gradient & Freeze-thaw \\
\hline Particle size $(\mathrm{nm})$ & $193 \pm 2.1$ & $178 \pm 5.5$ \\
PDI & $0.05 \pm 0.03$ & $0.12 \pm 0.07$ \\
EE $(\%)$ & $35.2 \pm 5$ & $51 \pm 7$ \\
\hline
\end{tabular}
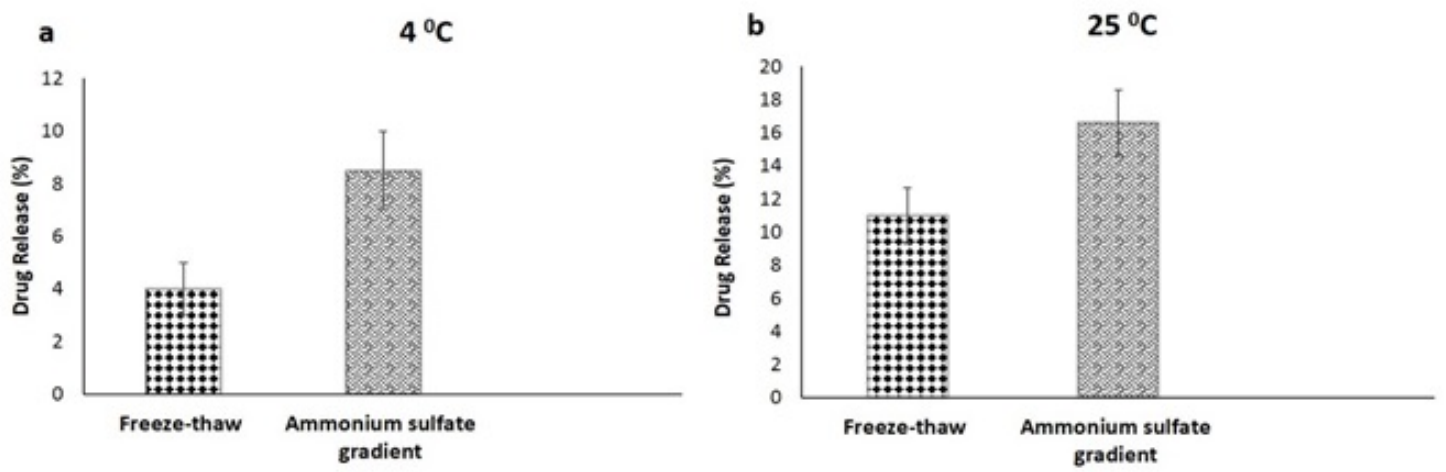

Fig. 2. The stability results of each formulation at $4^{\circ} \mathrm{C}$ (a) and $25^{\circ} \mathrm{C}$ (b) for 6 months
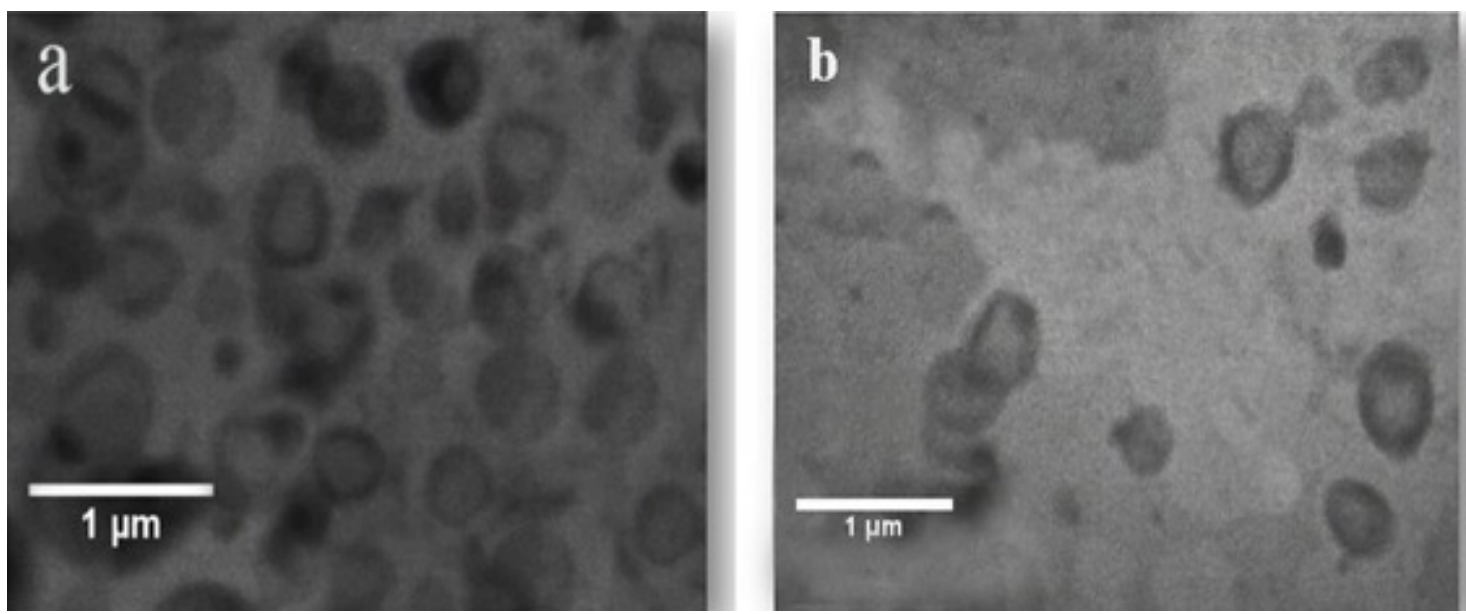

Fig. 3. TEM images of negatively stained liposome nanoparticles. Liposomal vancomycin prepared by ammonium sulfate method (a); Liposomal vancomycin prepared by freeze-thaw method (b). 
Table 2. Minimum inhibitory concentration (MIC) $(\mu \mathrm{g} / \mathrm{mL})$ for free and liposomal vancomycin

\begin{tabular}{ccc}
\hline Bacterial name & Free vancomycin & Liposomal Vancomycin \\
\hline S. aureus (ATCC 25923) & $<0.7$ & $<0.7$ \\
MRSA & $\leq 3$ & $\leq 1.5$ \\
\hline
\end{tabular}

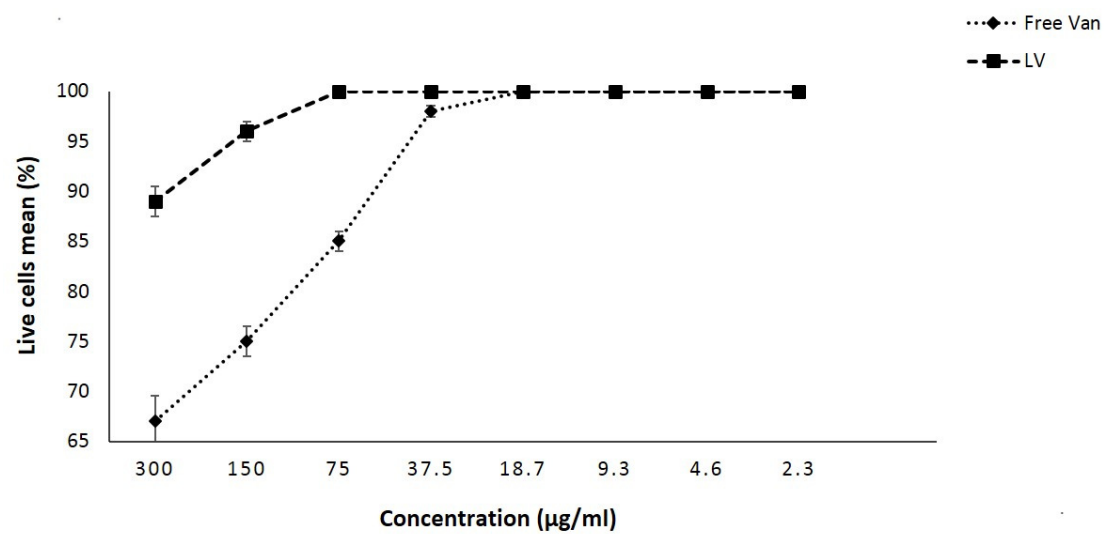

Fig. 4. Cytotoxicity assay of free vancomycin and liposomal vancomycin (LV) after incubation with human epidermoid carcinoma epithelial cell line (A431) at different concentration.

MIC and $3 \times$ MIC dosage, respectively.

\section{MRSA mortality and mice treatment}

The results showed that mice receiving $10^{9}, 10^{8}$, and $10^{7}$ CFU died after 2, 3, and 4 days, respectively (Fig. 5); MRSA was isolated from liver, spleen, and blood of all those mice, which was an indication of sepsis. Mice that were not exposed to infection stayed alive. According to these findings, $10^{7} \mathrm{CFU}$ of MRSA was used in subsequent experiments to infect all groups of mice. Next, the effect of each formulation on MRSA infection was evaluated. All mice treated with liposomal vancomycin and free vancomycin were treated after 14 days compared to the first day of MRSA infection ( $\mathrm{p}<0.05)$, among which the more effective result belonged to the mice treated with liposomal vancomycin in 9 and 14 days $(\mathrm{p}<0.05)$ (Table 3$)$.

\section{Discussion}

MRSA is the most common pathogen isolated from skin infections postsurgery (16). Vancomycin is used as an effective antibiotic against MRSA strain and is delivered

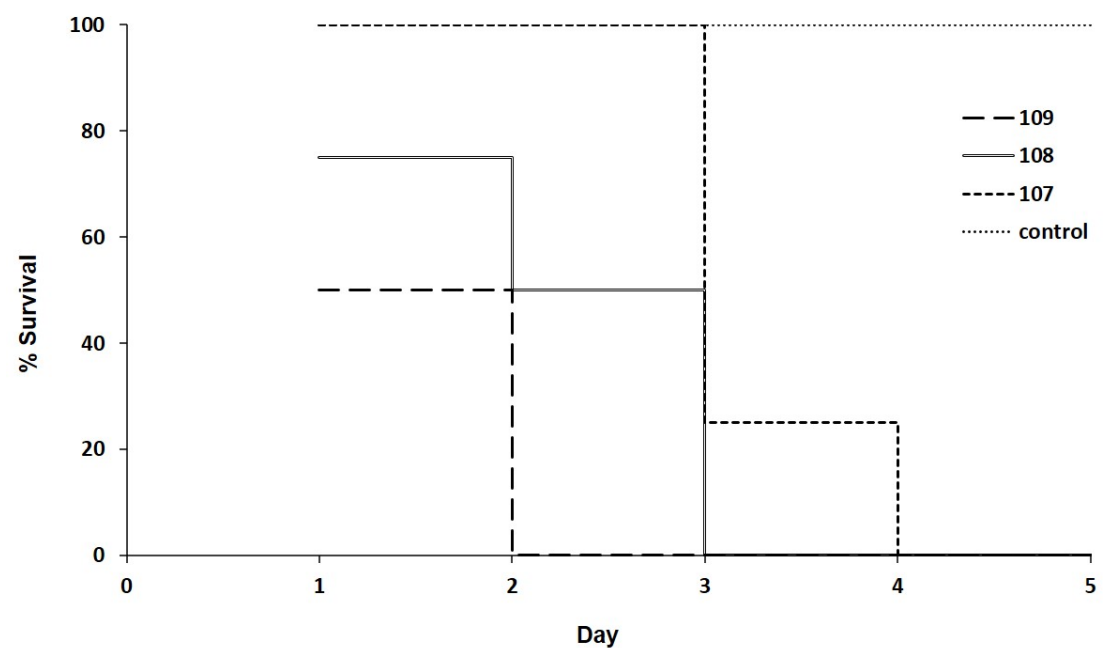

Fig. 5. Mortality in mice was caused by MRSA wound infection. A region of the skin was excised by biopsy punch and the wound was infected through different CFU counts of MRSA, including $10^{7}, 10^{8}$ and $10^{9}$; then, survival of mice on a daily basis was observed and detected. Mice receiving $10^{9}, 10^{8}$, and $10^{7} \mathrm{CFU}$ died after 2,3 , and 4 days, respectively.

Table 3. The bacterial counts (CFU/g) of mice skin section after treatment

\begin{tabular}{cccc}
\hline Day & MRSA & Free vancomycin +MRSA & Liposomal vancomycin +MRSA \\
\hline 0 & $3 \times 10^{7}$ & $5.5 \times 10^{7}$ & $5.5 \times 10^{7}$ \\
4 & Fatal & $5.7 \times 10^{7} \pm 4.4 \times 10^{7}$ & $1.8 \times 10^{7} \pm 2.7 \times 10^{7}$ \\
9 & Fatal & $4.3 \times 10^{5} \pm 2.8 \times 10^{5}$ & $3.2 \times 10^{4} \pm 2.1 \times 10^{4}$ \\
14 & Fatal & $295 \pm 22$ & $105 \pm 19$ \\
\hline
\end{tabular}


orally or parenterally (17). However, due to high adverse side effects, its use is limited. In addition, clinical failure has occurred due to poor penetration into eukaryotic cells and the emergence of vancomycin resistant strains $(4,18)$. In this study, vancomycin nanocarriers was developed as a new platform to be efficiently and safely applied for vancomycin topical administration to treat skin infections and surgical site infections. For this purpose, liposomes were used as antimicrobial carriers. Liposomes have several attractive features: lipid bilayer structure, mimicking bacterial membranes, and facilitating fusion between the two (19). As a result, an encapsulated antibiotic can be released for a longer time around or in the interior of a bacterial cell (20), leading to an increase in the local drug concentrations. Moreover, Manconi et al showed that the use of liposomes causes an enhancement in the drug transmission process through the skin and that the liposomes have the ability to penetrate the sublayers and to transport more effective drugs by lipid exchanging with skin lipids (21). It has also been shown that the use of phospholipids is important in the effective penetration of the drug through the skin and penetrates into the deep layers of skin under stratum corneum. Freeze-thaw and ammonium sulfate gradient methods were used to prepare liposomal vancomycin. A high entrapment of vancomycin was obtained by the mentioned methods ( $51 \%$ and $35.2 \%$, respectively), compared to other studies $(22,23)$, and it was higher in the freeze-thaw method than in ammonium sulfate gradient method. The higher encapsulation efficiency in the freeze-thaw method might have been due to the structural change towards a less multilamellar structure (24). However, in the study by Liu et al (2014), the rate of encapsulation efficiency in liposome was similar to the present study and applied as a potential delivery system to decrease nephrotoxicity (12). Negatively stained transmission electron microscopy and DLS measurement showed that both the liposomal formulations were less than $200 \mathrm{~nm}$ (Fig. 3 and Table 1). Moreover, in this study, the stability of both liposomal formulations at $4^{\circ} \mathrm{C}$ and $25^{\circ} \mathrm{C}$ was evaluated during 6 months (Figs 2a, b). The results showed that liposomal formulation prepared by freezethaw method was more stable than liposomal formulation prepared by ammonium sulfate gradient method, which may be related to the lamellarity, while increasing the number of layers decreases the drug release. Liposomes that were encapsulated using ammonium sulfate gradient method were more unstable, and this instability may be due to the crystal structure, and these crystals damage unilamellar structures and increase the vesicle leakage. In vitro study has shown that liposomal vancomycin had approximate one-fold improved MIC values for MRSA strain compared to free vancomycin (Table 2). In a study by Sande et al, 2 different types of liposomal vancomycin were synthesized with various lipids. The MIC results indicated that using liposomal vancomycin was twice as effective compared to free vancomycin. The results were different from those obtained in the in vitro tests from the present study which can be due to the type and percentage of lipids (25). In the present study, the results of the semi-quantitative analysis of CFU from the skin mice indicated that the number of bacteria was significantly reduced after 14 days compared to the first day $(\mathrm{p}<0.05)$. Although using free vancomycin was effective against MRSA in the mouse model, liposomal vancomycin was more effective than free vancomycin $(p<0.05)$, which was due to the large size of vancomycin molecule and less penetration to the skin tissue. The use of liposomal vancomycin has several advantages, including minimizing the emergence of drug resistance, reducing the dose for antimicrobial agent, more effective drug penetration, reduction of drug toxicity, and releasing a large amount of drug locally around the bacteria, which resulted in antibacterial effect (26). In this study, maximum effect was observed by using the minimum dose of vancomycin. Therefore, this reaction could turn bacterial killing capability from MIC into MBC dose. In this study, the inhibition dose of liposomal vancomycin was more effective than free vancomycin and it was found that the liposomal form has no toxicity up to 6 times MIC dosage on human epidermis cells. A study conducted by Abdelkader showed that the use of liposomal form resulted in continuous releasing of antibiotics (27). The release of vancomycin from the liposomal form continues with long releasing properties, so that the MRSA strain will be in touch with vancomycin during this period and the bactericidal effect is enhanced. In general, the difference between the free drugs with the entrapment drug in the upper doses is not known, because the use of the high dose of antibiotics leads to the destruction of a bacterial. However, the aforementioned problem has been solved using the liposomal form in low dose. Moreover, using liposomal form leads to a better penetration of vancomycin into eukaryotes cell compared to free vancomycin; thus, it is able to kill all intracellular and extracellular MRSA (18).

\section{Conclusion}

In this study, the synthesized nanoliposome was used as an effective and safe drug on the MRSA strain. In addition, to reduce drug toxicity, fewer doses of the drug can be used when using nanoliposome. It is also our belief that the use of synthesized nanoliposome leads to less resistance in bacteria. Therefore, this treatment can be used as an effective therapy.

\section{Conflict of Interests}

The authors declare that they have no competing interests.

\section{References}

1. Kalita S, Devi B, Kandimalla R, Sharma KK, Sharma A, Kalita K, et al. Chloramphenicol encapsulated in poly- $\varepsilon$-caprolactone-pluronic composite: nanoparticles for treatment of MRSA-infected burn wounds. Int J Mol Med. 2015;10:2971.

2. Schubert M, Kämpf D, Jatzwauk L, Kynast F, Stein A, Strasser R, et al. Prevalence and predictors of MRSA carriage among employees in a non-outbreak setting: a cross-sectional study in an acute care hospital. J Occup Med Toxicol. 2019;14(1):7.

3. Now MM, Common BSAS. Skin and soft-tissue infections: classifying and treating a spectrum. Clev Clin j med. 2012;79(1):57.

4. Boswihi SS, Udo EE. Methicillin-resistant Staphylococcus aureus: An update on the epidemiology, treatment options and infection control. Curr Med Res Pract. 2018.

5. Bruniera F, Ferreira F, Saviolli L, Bacci M, Feder D, da Luz Goncalves Pedreira M, et al. The use of vancomycin with its therapeutic and adverse effects: a review. Eur Rev Med Pharmacol Sci. 
2015;19(4):694-700.

6. Berger A, Oster G, Edelsberg J, Huang X, Weber DJ. Initial treatment failure in patients with complicated skin and skin structure infections. Surg Infect. 2013;14(3):304-12.

7. Banerjee K, Banerjee S, Das S, Mandal M. Probing the potential of apigenin liposomes in enhancing bacterial membrane perturbation and integrity loss. J. Colloid Interface Sci. 2015;453:48-59.

8. Shapiro AB, Newman J, Goteti K, Beaudoin ME, Harrison R, Hopkins $\mathrm{S}$, et al. Improvement of the pharmacokinetics and in vivo antibacterial efficacy of a novel type IIa topoisomerase inhibitor by formulation in liposomes. Antimicrob Agents Chemother. 2013;57(10):4816-24.

9. Ferreira M, Bernardo L, Neves L, Campos M, Lamaro-Cardoso J, André M. Virulence profile and genetic variability of Staphylococcus aureus isolated from artisanal cheese. J Dairy Sci. 2016;99(11):858997.

10. Shore AC, Deasy EC, Slickers P, Brennan G, O'Connell B, Monecke $\mathrm{S}$, et al. Detection of staphylococcal cassette chromosome mec type XI carrying highly divergent mecA, mecI, mecR1, blaZ, and ccr genes in human clinical isolates of clonal complex 130 methicillin-resistant Staphylococcus aureus. Antimicrob Agents Chemother. 2011;55(8):3765-73.

11. Clinical, Institute LS. Performance standards for antimicrobial susceptibility testing. Document M100-S15. Clinical and Laboratory Standards Institute Wayne, PA; 2016

12. Liu J, Wang Z, Li F, Gao J, Wang L, Huang GJajops. Liposomes for systematic delivery of vancomycin hydrochloride to decrease nephrotoxicity: Characterization and evaluation. Asian J Pharm Sci. 2015;10(3):212-22.

13. Labella C, Lelario F, Bufo SA, Musto M, Freschi P, Cosentino C. Optimization and validation of a chromatographic method for quantification of lysozyme in jenny milk. J Food Nutr Res. 2016;55(3).

14. Tseng CW, Sanchez-Martinez M, Arruda A, Liu GY. Subcutaneous infection of methicillin resistant Staphylococcus aureus (MRSA). J Visual Experim. 2011;48.

15. Tkalčević VI, Čužić S, Parnham MJ, Pašalić I, Brajša K. Differential evaluation of excisional non-occluded wound healing in $\mathrm{db} / \mathrm{db}$ mice. J Toxicol Pathol. 2009;37(2):183-92.

16. Misteli H, Rosenthal R, Oertli D, Marti W, Widmer A, Weber WP. Spectrum of pathogens in surgical site infections at a Swiss university hospital. Swiss Med Wkly. 2011;141(0304).

17. Gomes D, Pereira M, Bettencourt AF. Osteomyelitis: an overview of antimicrobial therapy. Braz J Pharm Sci. 2013;49(1):13-27.

18. Surewaard BG, Deniset JF, Zemp FJ, Amrein M, Otto M, Conly J, et al. Identification and treatment of the Staphylococcus aureus reservoir in vivo. J Exp Med. 2016:jem. 20160334.

19. Rukavina Z, Vanić ŽJP. Current trends in development of liposomes for targeting bacterial biofilms. Pharmaceutics. 2016;8(2):18.

20. Sharma A, Kumar Arya D, Dua M, Chhatwal GS, Johri AK. Nanotechnology for targeted drug delivery to combat antibiotic resistance. Taylor \& Francis; 2012.

21. Manconi M, Caddeo C, Sinico C, Valenti D, Mostallino MC, Biggio G, et al. Ex vivo skin delivery of diclofenac by transcutol containing liposomes and suggested mechanism of vesicle-skin interaction. Eur J Pharm Biopharm. 2011;78(1):27-35.

22. Muppidi K, Pumerantz AS, Wang J, Betageri GJIp. Development and stability studies of novel liposomal vancomycin formulations. ISRN Pharmaceutics. 2012;2012.

23. Pumerantz A, Muppidi K, Agnihotri S, Guerra C, Venketaraman V, Wang J, et al. Preparation of liposomal vancomycin and intracellular killing of meticillin-resistant Staphylococcus aureus (MRSA). J Am Coll Clin Pharm. 2011;37(2):140-4.

24. Sriwongsitanont S, Ueno MJTOCSJ. Effect of freeze-thawing process on the size and lamellarity of peg-lipid liposomes. Open Colloid Sci J. 2010;4(1).

25. Sande L, Sanchez M, Montes J, Wolf AJ, Morgan MA, Omri A, et al. Liposomal encapsulation of vancomycin improves killing of methicillin-resistant Staphylococcus aureus in a murine infection model. J Antimicrob Chemother. 2012;67(9):2191-4.

26. Nguyen HM, Graber CJJJoac. Limitations of antibiotic options for invasive infections caused by methicillin-resistant Staphylococcus aureus: is combination therapy the answer? J Antimicrob Chemother. 2009;65(1):24-36.

27. Abdelkader H, G Alany R. Controlled and continuous release ocular drug delivery systems: pros and cons. Curr Drug Deliv. 2012;9(4):42130 . 